\begin{tabular}{|c|l|}
\hline Title & Systematic investigations of transient response of nuclear spins in the presence of polarized electrons \\
\hline Author(s) & Rasly, Mohmoud; Lin, Zhichao; Uemura, Tetsuya \\
\hline Citation & $\begin{array}{l}\text { Physical Review B, 96(18), 184415 } \\
\text { https://doi.org/10.1103/PhysRevB.96.184415 }\end{array}$ \\
\hline Issue Date & 2017-11-13 \\
\hline Doc URL & http://hdl.handle.net/2115/67835 \\
\hline Rights & ○2017 A merican Physical Society \\
\hline Type & article \\
\hline File Information & PhysRevB.96.184415.pdf \\
\hline
\end{tabular}

Instructions for use 


\title{
Systematic investigations of transient response of nuclear spins in the presence of polarized electrons
}

\author{
Mohmoud Rasly, Zhichao Lin, and Tetsuya Uemura* \\ Division of Electronics for Informatics, Hokkaido University, Sapporo 060-0814, Japan \\ (Received 5 August 2017; revised manuscript received 25 October 2017; published 13 November 2017)
}

\begin{abstract}
We electrically probed the transient response of nuclear spins in an $n$-GaAs channel by performing Hanle signal and spin-valve signal measurements on an all-electrical spin-injection device having a half-metallic spin source of $\mathrm{Co}_{2} \mathrm{MnSi}$. Furthermore, we simulated the Hanle and spin-valve signals by using the time evolution of nuclear-spin polarization under the presence of polarized electron spins by taking both $T_{1 \mathrm{e}}$ and $T_{1}$ into consideration, where $T_{1 \mathrm{e}}^{-1}$ is the polarization rate of nuclear spins through the transfer of angular momentum from polarized electron spins and $T_{1}^{-1}$ is the depolarization rate of nuclear spins through the interaction with the lattice. The simulation results reproduced our experimental results on all the nuclear-spin-related phenomena appearing in the Hanle and spin-valve signals at different measurement conditions, providing quantitative explanation for the transient response of nuclear spins in GaAs to a change in magnetic fields and an estimate of the time scales of $T_{1 \mathrm{e}}$ and $T_{1}$. These experimental and simulated results will deepen the understanding of nuclear-spin dynamics in semiconductors.
\end{abstract}

DOI: 10.1103/PhysRevB.96.184415

\section{INTRODUCTION}

Nuclear-spin systems are ideal for implementing quantum bits (qubits) because of their extremely long coherence time. Nevertheless, it is difficult to polarize the nuclear spins of a semiconductor and to detect the nuclear-spin states because of their tiny magnetic moment. A possible solution is using dynamic nuclear polarization (DNP), in which the nuclear spins are polarized by polarized electron spins through the hyperfine interaction. The polarized nuclear spins, in turn, affect electron spins through an effective magnetic field or Overhauser field $\left(\mathbf{B}_{\mathbf{n}}\right)$. This interaction dramatically increases the signal intensity available for detection of the nuclear-spin states compared with pick-up-coil detection. Several methods to create and detect nuclear spins through the hyperfine interaction have been demonstrated by using optically- [1-13] or electrically [14-25]-created electron spins.

Electrical spin injection using ferromagnetic electrodes as a source of electron spins is the most recent proposal for an electrical DNP [16,18-25]. In such a system, a highly efficient spin source is essential for an effective DNP. A Co-based Heusler alloy is an excellent ferromagnetic candidate for spintronic devices, including magnetic tunnel junctions (MTJs) [26-35], giant magnetoresistance devices [36-42], and for spin injection into semiconductors [43-48], due to its complete spin polarization at the Fermi level [49-51]. We recently reported high tunneling magnetoresistance ratios of up to $1995 \%$ at $4.2 \mathrm{~K}$ and up to $354 \%$ at $290 \mathrm{~K}$ in MTJs having $\mathrm{Mn}$-rich $\mathrm{Co}_{2} \mathrm{MnSi}$ (CMS) electrodes [32], and found ratios of $2610 \%$ at $4.2 \mathrm{~K}$ and $429 \%$ at $290 \mathrm{~K}$ in Mn-rich $\mathrm{Co}_{2}(\mathrm{Mn}, \mathrm{Fe}) \mathrm{Si}$ MTJs [33,34], demonstrating a high spin polarization of CMS and CMFS. Moreover, we achieved highly efficient spin injection into a GaAs channel by using Mn-rich CMS as a spin source $[23,46]$. Polarization of electron spins in GaAs reached $52 \%$ at $4.2 \mathrm{~K}$ [46], which promotes efficient DNP.

The dynamics of nuclear spins in GaAs has been investigated at various measurement conditions such as temperature

\footnotetext{
*uemura@ist.hokudai.ac.jp
}

and magnetic field strength [9], the excitation energy of photons $[5,12]$, and also at different impurity concentrations for GaAs itself [11]. However, the time evolution of dynamically polarized nuclear spins is not fully understood yet, and it is still controversial. Basically, the nuclear-spin system interacts with the electron-spin system through the hyperfine interaction at a polarization rate of $1 / T_{1 \mathrm{e}}$ and also with the lattice at a depolarization rate of $1 / T_{1}$ [52]. Therefore, sweeping the external magnetic field on a time scale faster than $T_{1 \mathrm{e}}$ and/or $T_{1}$ causes the nuclear spins to behave in a complicated manner. The quantitative analyses of the DNP thus have mostly been limited to steady-state circumstances [20], where the change in the external magnetic field is relatively slow.

Previously, we experimentally investigated the behavior of nuclear spins under transient-state circumstances, in which the magnetic field was swept faster than the time scale of $T_{1 \mathrm{e}}$ or $T_{1}$, in an all-electrical spin-injection device, and observed a clear transient response of nuclear spins against the change in the magnetic field $[23,24]$. Soon afterward, we analyzed this behavior using the concept of nuclear-spin temperature for the case with and without nuclear magnetic resonance [53,54]. However, the simulation and the experiment were on a fixed time scale. Moreover, our previous simulation model did not include the spin-lattice relaxation mechanism, which means that the description of the nuclear-spin states was incomplete especially near zero-magnetic-field regions. The lack of such information presents a difficulty in achieving the precise control of nuclear spins.

The purpose of the present study was to clarify the transient behavior of nuclear spins in semiconductor in a quantitative manner. To do this, we systematically investigated the dependencies of nuclear-spin polarization on the initialization time for the DNP and the sweep rates of the magnetic field through the Hanle signal and spin-valve signal measurements made on an all-electrical lateral spin-injection device having $\mathrm{Co}_{2} \mathrm{MnSi} / \mathrm{CoFe} / n$-GaAs Schottky tunnel junctions. In addition, we simulated all the nuclear-spin-related phenomena appearing in the Hanle/spin-valve signals using the time evolution of nuclear field, taking both the rate of polarization $\left(1 / T_{1 \mathrm{e}}\right)$ and rate of depolarization $\left(1 / T_{1}\right)$ into consideration. 
(a)

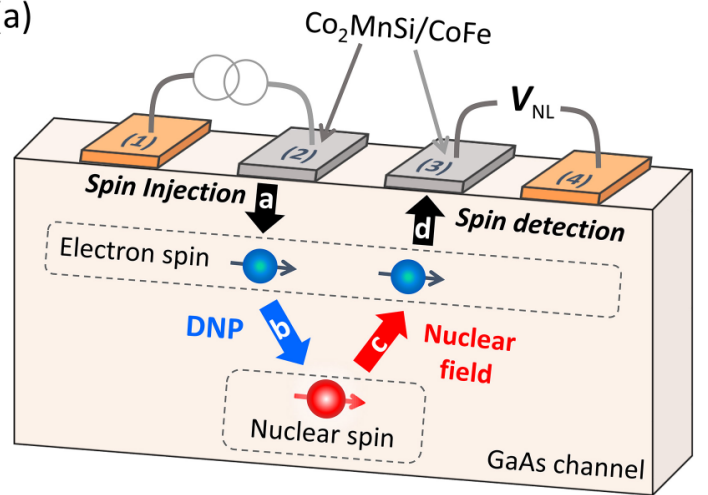

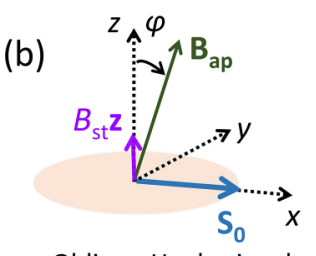

Oblique Hanle signal

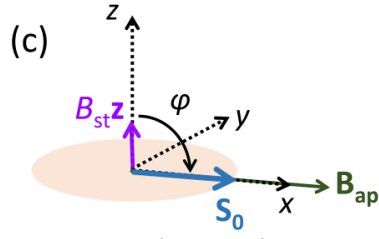

Spin valve signal
FIG. 1. (a) Schematic device structure of an all-electrical spininjection device and circuit configuration for nonlocal Hanle and spinvalve measurements, (b) measurement configurations for an oblique Hanle signal, and (c) for a spin-valve signal, where $\mathbf{B}_{\text {ap }}$ is the applied magnetic field, $B_{\mathrm{st}}$ is the stray field from the ferromagnetic electrodes, $\mathbf{S}_{\mathbf{0}}$ is the injected electron spin, and $\varphi$ is the oblique angle from the $z$ axis in the $x-z$ plane.

This paper is organized as follows. In Sec. II, we describe an experiment including the measurement methodology and a method of simulating a transient response of $\mathbf{B}_{\mathbf{n}}$ in GaAs. Section III contains our experimental and simulation results on the nuclear-spin-related phenomena appearing in the Hanle and spin-valve signals. We analyze the transient response of nuclear spins to a change in a magnetic field, showing precise control of the strength of $\mathbf{B}_{\mathbf{n}}$ along with its direction in space. Section IV summarizes our results and concludes the paper.

\section{EXPERIMENTAL AND SIMULATION MODEL}

\section{A. Experimental and measurement methodology}

A lateral spin-transport device having $\mathrm{Co}_{2} \mathrm{MnSi} / \mathrm{CoFe} /$ GaAs heterojunctions was fabricated [Fig. 1(a)]. The layer structure and device geometry are identical to the ones presented in Ref. [23]. The device operation is as follows: The spin-polarized electrons are generated in the GaAs by spin injection from ferromagnet $\left(\mathrm{Co}_{2} \mathrm{MnSi}\right)$ into $\mathrm{GaAs}$ [see pass $a$ in Fig. 1(a)]. Those polarized electron spins exchange angular momentum with the nuclear spins through the hyperfine interaction (pass $b$ ), leading to the DNP. The polarized nuclear spins, in turn, affect back on the electron spins by an effective magnetic field or Overhauser field $\left(\mathbf{B}_{\mathbf{n}}\right)$ (pass $c$ ). Then, the nuclear-spin states can be read out by detecting the Larmor precession of the electron spin by measuring the change in the nonlocal voltage (pass $d$ ).

We measured two kinds of signal at $4.2 \mathrm{~K}$, Hanle and spin-valve signals, in a four-terminal nonlocal geometry in which the nonlocal voltage $\left(V_{\mathrm{NL}}\right)$ between contact 3 and contact 4 was measured under a constant bias current $I_{\text {bias }}$ supplied between contact 1 and contact 2 . The main difference between the oblique Hanle signal measurement and the spinvalve signal measurement is the direction of applied magnetic field $\mathbf{B}_{\mathbf{a p}}$, as clarified in Figs. 1(b) and 1(c), respectively. For the oblique Hanle signal measurement, $\mathbf{B}_{\text {ap }}$ was applied along an oblique direction $\varphi=8^{\circ}$ from the $z$ axis in the $x$-z plane-i.e., $\mathbf{B}_{\mathrm{ap}} \equiv B_{\mathrm{ap}}(\mathbf{x} \sin \varphi+\mathbf{z} \cos \varphi)$, where $B_{\text {ap }}$ is the strength of $\mathbf{B}_{\text {ap }}$ (negative $B_{\text {ap }}$ means that the direction of $\mathbf{B}_{\text {ap }}$ is reversed), and $\mathbf{x}$ and $\mathbf{z}$ are unit vectors along the $x$ - and $z$-axis directions, respectively, in order to detect the Hanle precession of electron spins. Because the easy axis of magnetization for the $\mathrm{Co}_{2} \mathrm{MnSi} / \mathrm{CoFe}$ bilayer was parallel to the $x$ axis, the injected electron spins $\mathbf{S}_{\mathbf{0}}$ pointed toward the $x$ axis. Thus, in contrast to the conventional Hanle signal measurement, $\mathbf{B}_{\mathbf{a p}}$ has a component parallel to the injected electron spins. The nonzero component of $\mathbf{B}_{\mathrm{ap}} \cdot \mathrm{S}_{\mathbf{0}}$ allows the nuclear spins to be polarized along the magnetic field through the hyperfine interaction, as will be shown in the next section. In order to study the transient response of nuclear spins to a change in $\mathbf{B}_{\text {ap }}$, the magnetic field was initially held at $B_{\text {ap }}=+48 \mathrm{mT}$ for a hold time of $t_{\text {hold }}$, and then it was swept from $B_{\text {ap }}=+48$ to $-48 \mathrm{mT}$ at a certain sweep rate $(r)$ which was selected to be faster than the one required to bring the nuclear spins to their equilibrium state. $I_{\text {bias }}$ was set to $-70 \mu \mathrm{A}$, under which electron spins were injected from contact 2 into the GaAs with the polarization at the ferromagnet/GaAs interface of $12.8 \%$ [23].

For the spin-valve signal measurement, $\mathbf{B}_{\mathbf{a p}}$ was applied along the $x$ axis, parallel to the easy-axis direction of magnetization for the $\mathrm{Co}_{2} \mathrm{MnSi} / \mathrm{CoFe}$ bilayer, i.e., $\varphi=90^{\circ}$ in $\mathbf{B}_{\mathrm{ap}}=B_{\mathrm{ap}}(\mathbf{x} \sin \varphi+\mathbf{z} \cos \varphi)$, in order to detect the change of $V_{\mathrm{NL}}$ when the magnetization direction of the injector and detector is switched between parallel and antiparallel states. $I_{\text {bias }}$ was set to $-40 \mu \mathrm{A}$. We used two different schemes of sweeping $B_{\text {ap }}$. In the first scheme, similarly to the conventional spin-valve measurement, $B_{\text {ap }}$ was swept from 0 to $-40 \mathrm{mT}$; then it was swept back from -40 to $+40 \mathrm{mT}$, and finally from $+40 \mathrm{mT}$ to zero at a fixed sweep rate $r$ of $0.5 \mathrm{mT} / \mathrm{s}$. In the second scheme, we stopped the sweeping of $\mathbf{B}_{\mathbf{a p}}$ just after $\mathbf{B}_{\text {ap }}$ returned to zero at $t=160 \mathrm{~s}$ in order to test the transient response of $\mathbf{B}_{\mathbf{n}}$ at $B_{\text {ap }}=0$. The detailed conditions about the sweep of the magnetic field in both the oblique Hanle and spin-valve measurements are summarized in Appendix A.

\section{B. Simulation model}

We simulated the oblique Hanle signals and spin-valve signals measured under the above conditions by considering the time evolution of the nuclear field. As described in the Introduction, the nuclear-spin system interacts with the electron-spin system through the hyperfine interaction at a polarization rate of $1 / T_{1 \mathrm{e}}$ and also with the lattice at a depolarization rate of $1 / T_{1} . T_{1}$ varies with the magnetic field B experienced by nuclei, and it is given by $[18,52]$

$$
T_{1}=T_{1 \mathrm{e}}\left(\frac{\mathbf{B}^{2}}{\xi B_{\mathrm{L}}^{2}}\right),
$$

where $\xi$ is a numerical coefficient that depends on the nature of the spin-spin interactions, and $B_{\mathrm{L}}$ is the local dipolar field. 
When the direction and strength of $\mathbf{B}$ change, the direction of $\mathbf{B}_{\mathbf{n}}$ changes in response quickly because of its adiabatic process, while the strength of $\mathbf{B}_{\mathbf{n}}$ changes slowly on a time scale of $\left(1 / T_{1 \mathrm{e}}+1 / T_{1}\right)^{-1}$ because of a nonadiabatic process through the interactions with the electron spins or the lattice. This behavior can be described in terms of the time evolution of the nuclear field $\mathbf{B}_{\mathbf{n}}$ in the DNP using the following expressions:

$\mathbf{B}_{\mathbf{n}}(t)=B_{\mathrm{n}}(t) \frac{\mathbf{B}}{|\mathbf{B}|}, \frac{d}{d t} B_{\mathrm{n}}(t)=-\left(\frac{1}{T_{1 \mathrm{e}}}+\frac{1}{T_{1}}\right)\left(B_{\mathrm{n}}(t)-B_{\mathrm{n} 0}\right)$,

where $B_{\mathrm{n} 0}$ is the static solution, which is given by [52]

$$
B_{\mathrm{n} 0}=f b_{n} \frac{\mathbf{B} \cdot \mathbf{S}}{\mathbf{B}^{2}+\xi B_{L}^{2}}|\mathbf{B}|,
$$

where $f(\leqslant 1)$ is the leakage factor, $b_{\mathrm{n}}$ is the effective field due to the polarization of nuclear spins, which takes the negative value of $-17 \mathrm{~T}$ in GaAs for the theoretical ideal case, and $\mathbf{S}$ is the average electron spin $(|\mathbf{S}|=1 / 2$ corresponds to $P_{\mathrm{GaAs}}=100 \%$ ). The derivation of Eqs. (1)-(3) will be given in Appendix B. In addition to $\mathbf{B}_{\text {ap }}$, we assumed a constant stray field $B_{\mathrm{st}}=1.0 \mathrm{mT}$ from the ferromagnetic electrodes, which pointed along the $z$-axis direction in the GaAs channel, as part of the external field in our simulation. Accordingly, the total external magnetic field $\mathbf{B}$ is given by

$$
\mathbf{B}=\mathbf{B}_{\mathrm{ap}}+B_{\mathrm{st}} \mathbf{z}=B_{\mathrm{ap}}(\mathbf{x} \sin \varphi+\mathbf{z} \cos \varphi)+B_{\mathrm{st}} \mathbf{z} .
$$

Since electron spins experience $\mathbf{B}+\mathbf{B}_{\mathbf{n}}, \mathbf{S}$ precesses along $\mathbf{B}+\mathbf{B}_{\mathbf{n}}$, whose direction is along $+\mathbf{B}$ or $-\mathbf{B}$, because $\mathbf{B}$ and $\mathbf{B}_{\mathbf{n}}$ are either parallel or antiparallel to each other. This precession changes only the component of $\mathbf{S}$ perpendicular to $\mathbf{B}$ and conserves the component of $\mathbf{S}$ parallel to $\mathbf{B}$. Thus, we approximated $\mathbf{B} \cdot \mathbf{S}$ in Eq. (3) by $\mathbf{B} \cdot \mathbf{S}_{\mathbf{0}}$ in our simulation.

The precession of electron spins along the total magnetic field of $\mathbf{B}+\mathbf{B}_{\mathbf{n}}$ results in a change in electron-spin polarization at detector contact 3 . Similarly to the conventional nonlocal Hanle signal, $V_{\mathrm{NL}}$ can be described as [55]

$V_{\mathrm{NL}}=A \int_{0}^{\infty} \frac{1}{\sqrt{4 \pi D t}} \exp \left(-\frac{d^{2}}{4 D t}\right) \cos \left(\omega_{\mathrm{L}} t\right) \exp \left(-\frac{t}{\tau_{s}}\right) d t$

where $A$ is a constant, $D$ is the diffusion constant, $d$ is the distance between contact 2 and contact $3, \tau_{\mathrm{s}}$ is the electronspin-relaxation time, and $\omega_{\mathrm{L}}=g_{\mathrm{e}} \mu_{\mathrm{B}} B_{\mathrm{z}} / \hbar$ is the Larmor frequency, where $B_{\mathrm{z}}$ is the $z$ component of $\mathbf{B}+\mathbf{B}_{\mathbf{n}}, g_{\mathrm{e}}$ is an electron $g$ factor $\left(g_{\mathrm{e}}=-0.44\right.$ for GaAs), $\mu_{\mathrm{B}}$ is the Bohr magneton, and $\hbar$ is the reduced Planck's constant.

Using Eqs. (1)-(5), we simulated the change in $V_{\mathrm{NL}}$ as a function of $B_{\text {ap }}$ or $t$. The parameters used in the simulation are listed in Table I. The values of $f$ and $T_{1 \mathrm{e}}$ were treated as fitting parameters.

\section{RESULTS AND DISCUSSION}

\section{A. Initialization-time dependence of oblique Hanle signals}

Figure 2 shows (a) measured oblique Hanle signals for $t_{\text {hold }}$ ranging from 25 to $150 \mathrm{~s}$ at a sweep rate of $r=0.7 \mathrm{mT} / \mathrm{s}$ and (b) the corresponding simulation result. Each single curve
TABLE I. Simulation parameters.

\begin{tabular}{lc}
\hline \hline Parameter & Value \\
\hline $2\left|\mathbf{S}_{\mathbf{0}}\right|$ & $12.8 \%$ \\
$l_{\mathrm{sf}}(\mu \mathrm{m})$ & 3 \\
$T_{1 \mathrm{e}}(\mathrm{s})$ & 450 \\
$f$ & 0.023 \\
$\sqrt{\xi} B_{\mathrm{L}}(\mathrm{mT})$ & 2.3 \\
$d(\mu \mathrm{m})$ & 0.5 \\
$\tau_{s}(\mathrm{~ns})$ & 20 \\
\hline \hline
\end{tabular}

represents the changes in $V_{\mathrm{NL}}$ while sweeping the magnetic field from $B_{\text {ap }}=+48$ to $-48 \mathrm{mT}$. The Hanle curves have three main peaks that appear regardless of the change in $t_{\text {hold }}$, and the side peaks for both the positive and negative $B_{\text {ap }}$ region shift to a higher magnetic field as $t_{\text {hold }}$ increases. (Hereafter, we call the side peak appearing at $B_{\text {ap }}>0$ a positive side peak and the one appearing at $B_{\text {ap }}<0$ a negative side peak.) As mentioned in Sec. II, $V_{\mathrm{NL}}$ gives the degree of electron-spin precession, and it becomes larger as $B_{\mathrm{Z}}$ becomes smaller. As will be shown later, $\mathbf{B}$ and $\mathbf{B}_{\mathrm{n}}$ cancel each other, i.e., $\mathbf{B}+\mathbf{B}_{\mathbf{n}}=\mathbf{0}$, at the side-peak position of $V_{\mathrm{NL}}$. This means that $\left|B_{\text {ap }}\right|$ at the side-peak position gives the strength of $\mathbf{B}_{\mathbf{n}}$, and the results for Figs. 2(a) and 2(b) suggest that the generated nuclear field increases as $t_{\text {hold }}$ increases.

The simulation results reproduced the experimental ones, which validate our simulation model. On the basis of the simulation results, we obtained $T_{1 \mathrm{e}}$ of $450 \mathrm{~s}$, which is a reasonable one for the DNP in a GaAs channel [18,19,23]. Furthermore, we plotted a vector diagram of the relative orientation of the injected electron spin $\mathbf{S}_{\mathbf{0}}$, magnetic field $\mathbf{B}$, and nuclear field $\mathbf{B}_{\mathbf{n}}$, along with a simulated oblique Hanle signal for $t_{\text {hold }}=100 \mathrm{~s}$ (Fig. 3). Here, the oblique Hanle curve can be divided into six main regions, or correspondingly states, as illustrated in Fig. 3. In the following, we will describe each state.

In state 1 , where $\mathbf{B}_{\text {ap }}$ was constant at $B_{\text {ap }}=+48 \mathrm{mT}$ for $0 \leqslant t \leqslant t_{\text {hold }}$, the nuclear spins were dynamically polarized through the hyperfine interaction by polarized electron spins. Since $b_{n}$ is negative in GaAs and $\mathbf{B} \cdot \mathbf{S}_{0}>0, \mathbf{B}_{\mathbf{n}}$ was generated antiparallel to $\mathbf{B}$ with the strength of $\mathbf{B}_{\mathbf{n}}$ smaller than that of $\mathbf{B}$. Although the strength of the total magnetic field
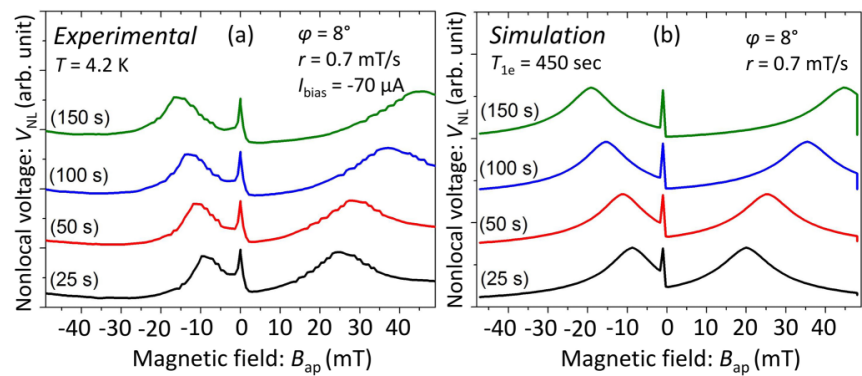

FIG. 2. (a) Experimental initialization-time ( $\left.t_{\text {hold }}\right)$ dependence of oblique Hanle signals for $t_{\text {hold }}$ ranging from 25 to $150 \mathrm{~s}$ at a sweep rate of $r=0.7 \mathrm{mT} / \mathrm{s}$ and (b) corresponding simulation result. Each single curve shows the change in $V_{\mathrm{NL}}$ while sweeping the magnetic field from $B_{\text {ap }}=+48$ to $-48 \mathrm{mT}$. 

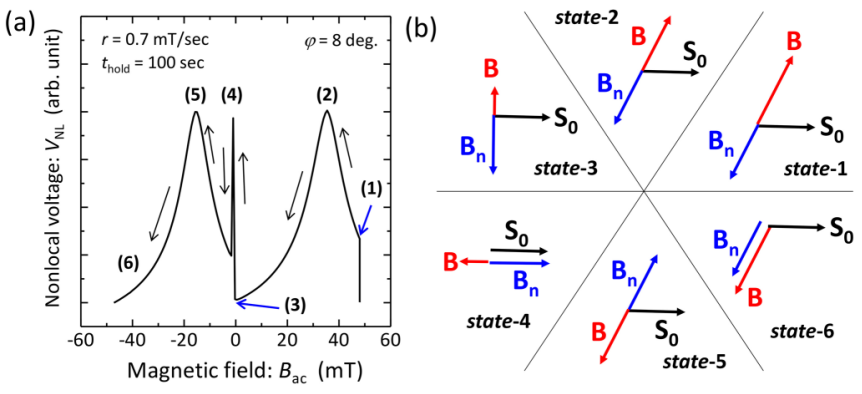

FIG. 3. (a) Simulation result for an oblique Hanle signal at a sweep rate $(r=0.7 \mathrm{mT} / \mathrm{s})$ faster than that one required to bring nuclear spins to their equilibrium conditions and (b) a corresponding schematic vector diagram for the relative orientation of the injected electron spin $\mathbf{S}_{\mathbf{0}}$, magnetic field $\mathbf{B}$, and nuclear field $\mathbf{B}_{\mathbf{n}}$.

$\left(\mathbf{B}+\mathbf{B}_{\mathbf{n}}\right)$ experienced by the electron spins was smaller than that of $\mathbf{B}$, it was still large enough to induce a strong electron-spin precession, resulting in a relatively low $V_{\mathrm{NL}}$, as shown in Fig. 3(a). In the sweeping process, $\mathbf{B}_{\text {ap }}$ was ramped down from $B_{\text {ap }}=48 \mathrm{mT}$ to 0 at a sweep rate $r$ of $0.7 \mathrm{mT} / \mathrm{s}$. This sweep rate was almost 280 times faster than that for the steady-state circumstances in Ref. [20], in which $r$ was on the order of $0.0025 \mathrm{mT} / \mathrm{s}$ for the nuclear spins to reach the equilibrium state. Since the time scale for the change in $B_{\mathrm{n}}$, which is given by $\left(1 / T_{1 \mathrm{e}}+1 / T_{1}\right)^{-1}$, was much slower than that of the change in $|\mathbf{B}|, B_{\mathrm{n}}$ decreased more gradually than $|\mathbf{B}|$. Thus, $\mathbf{B}+\mathbf{B}_{\mathbf{n}}$ decreased in strength, and hence $V_{\mathrm{NL}}$ increased until it reached a peak at $B_{\text {ap }}=35 \mathrm{mT}$ (state 2). In state 2, $\mathbf{B}_{\mathbf{n}}$ and $\mathbf{B}$ completely canceled each other. This means that $\mathbf{B}+\mathbf{B}_{\mathbf{n}}=\mathbf{0}$ and electron-spin precession was suppressed.

The magnitude of $\mathbf{B}_{\text {ap }}$ then continued to decrease, vanishing at $B_{\text {ap }}=0$ [state 3]. Thereafter, it increased in the negative direction. Because of the stray field of $B_{\mathrm{st}} \mathbf{z}, \mathbf{B}$ reversed direction by rotation in the $z-x$ plane from state 2 to state 5 through state- 3 and state 4 . When $\mathbf{B}$ changed its direction and strength, the direction of $\mathbf{B}_{\mathbf{n}}$ changed in response quickly because of its adiabatic process, while the strength of $\mathbf{B}_{\mathbf{n}}$ changed slowly on a time scale of $\left(1 / T_{1 \mathrm{e}}+1 / T_{1}\right)^{-1}$, as shown in Eq. (2). Thus, in response to the rotation of $\mathbf{B}, \mathbf{B}_{\mathbf{n}}$ also rotated, keeping an antiparallel relation with $\mathbf{B}$ until reaching state 5 . In state $3, \mathbf{B}_{\mathbf{n}}$ pointed perpendicular to $\mathbf{S}_{\mathbf{0}}$, leading to the electron spins experiencing a strong electron-spin precession, and $V_{\mathrm{NL}}$ took on a local minimum value. In state $4, \mathbf{B}$ and $\mathbf{B}_{\mathbf{n}}$ were parallel or antiparallel to $\mathbf{S}_{\mathbf{0}}$. Thus, no Hanle precession occurred and $V_{\mathrm{NL}}$ reached the central peak. As $B_{\text {ap }}$ decreased (i.e., increases in the negative side), $\mathbf{B}$ and $\mathbf{B}_{\mathbf{n}}$ canceled again in state 5 , resulting in a side peak of $V_{\mathrm{NL}}$ at $B_{\text {ap }}=-15 \mathrm{mT}$. Finally, in state 6 , since sufficient time for the nuclear spins to be negatively polarized had passed, $\mathbf{B}_{\mathbf{n}}$ turned parallel to $\mathbf{B}$, and electron spins experienced a strong magnetic field. After state $3, \mathbf{B} \cdot \mathbf{S}_{\mathbf{0}}$ turned negative, and the steady-state solution of the nuclear field $\mathbf{B}_{\mathbf{n} 0}$, defined by $B_{\mathrm{n} 0} \frac{\mathbf{B}}{|\mathbf{B}|}$, was parallel to $\mathbf{B}$ [see Eq. (4)]. Nevertheless, $\mathbf{B}_{\mathbf{n}}$ and $\mathbf{B}$ remained antiparallel until state 5 because the time constant given by $\left(T_{1 \mathrm{e}}^{-1}+T_{1}^{-1}\right)^{-1}$ was approximately $72 \mathrm{~s}$ or more, i.e., more than four times longer than the time taken for $\mathbf{B}$ to change from state 3 to state 5 .

Now let us turn back to explain the $t_{\text {hold }}$ dependence of the nuclear-spin polarization. Through the experimental or

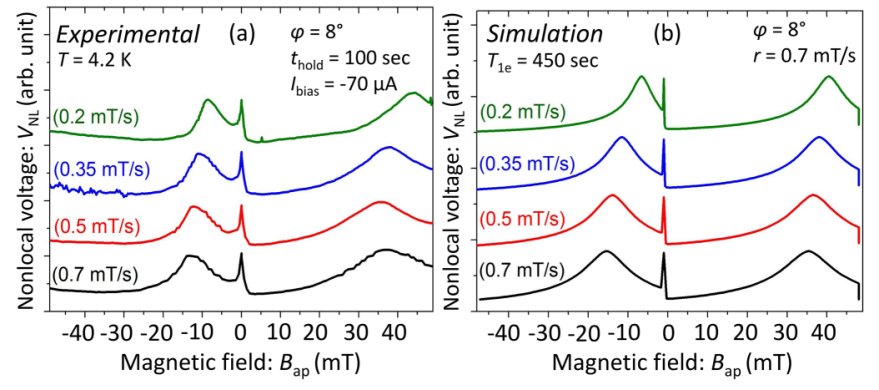

FIG. 4. (a) Experimental results of sweep rate $(r)$ dependence of oblique Hanle signals for $r$ ranging from 0.7 to $0.2 \mathrm{mT} / \mathrm{s}$ for $t_{\text {hold }}=100 \mathrm{~s}$ and (b) corresponding simulation result.

simulation results, the position of the satellite peak at $B_{\text {ap }}>0$ shifts to a higher magnetic field, which shows an almost exponential dependence on $t_{\text {hold }}$. This means that the strength of $\mathbf{B}_{\mathbf{n}}$ depends exponentially on $t_{\text {hold }}$. This is reasonable because Eq. (2) can be solved analytically when $0 \leqslant t \leqslant$ $t_{\text {hold }}$, during which $|\mathbf{B}|$ and $B_{\mathrm{n} 0}$ are constant, and $B_{\mathrm{n}}\left(t_{\text {hold }}\right)$ is given by

$$
B_{\mathrm{n}}\left(t_{\text {hold }}\right)=B_{\mathrm{n} 0}\left\{1-\exp \left[\left(\frac{1}{T_{1 \mathrm{e}}}+\frac{1}{T_{1}}\right) t_{\text {hold }}\right]\right\} .
$$

Thus, increasing $t_{\text {hold }}$ leads to an exponential increase in $B_{\mathrm{n}}$, and the magnitude of $\mathbf{B}_{\text {ap }}$ required to cancel $\mathbf{B}_{\mathbf{n}}$ also increases. Thus, the cancellation condition or peak point shifts to higher $B_{\text {ap }}$. The shift in the peak on the negative side to a larger $\left|B_{\text {ap }}\right|$ as $t_{\text {hold }}$ increases can be explained similarly. On the other hand, the central peak in state 4 does not shift even when changing $t_{\text {hold }}$. As we explained above, the central peak in state 4 originates from the suppression of electron-spin precession due to $\mathbf{B}+\mathbf{B}_{\mathbf{n}}$ being parallel to $\mathbf{S}_{\mathbf{0}}$. This contrasts with the origin of the side peaks, which is the cancellation of $\mathbf{B}$ and $\mathbf{B}_{\mathbf{n}}$. Therefore, in state 4 , the position of the peak is independent of the initialization process.

\section{B. Sweep-rate dependence of oblique Hanle signals}

Figure 4 shows (a) an experimental result for the sweep-rate dependence of oblique Hanle signals and (b) the corresponding simulation result. This experiment attempted to clarify the difference between the transient behavior and the static one for $\mathbf{B}_{\mathbf{n}}$. Each single curve shows a change in $V_{\mathrm{NL}}$ when the magnetic field was swept from $B_{\text {ap }}=+48$ to $-48 \mathrm{mT}$ at a rate $r=0.2,0.35,0.5$, and $0.7 \mathrm{mT} / \mathrm{s}$ while fixing $t_{\text {hold }}$ to $100 \mathrm{~s}$ in both the experiment and simulation. Here, $V_{\mathrm{NL}}$ has three main peaks regardless of the change in $r$. The position of the positive side peaks shifted to higher $B_{\text {ap }}$ as the sweep became slower, except for the experimental one with $r=0.7 \mathrm{mT} / \mathrm{s}$. On the other hand, the negative side peaks shifted to smaller $\left|B_{\text {ap }}\right|$ as the sweep became slower. The position of the central peaks did not change.

The $r$ dependence of the positive side peaks can be understood from the fact that the strength of the generated nuclear field increased as $r$ decreased, as explained in the previous section. The $r$ dependence of the negative side peaks, on the other hand, originated mainly from the difference between the transient state and steady state. As $r$ decreased, 


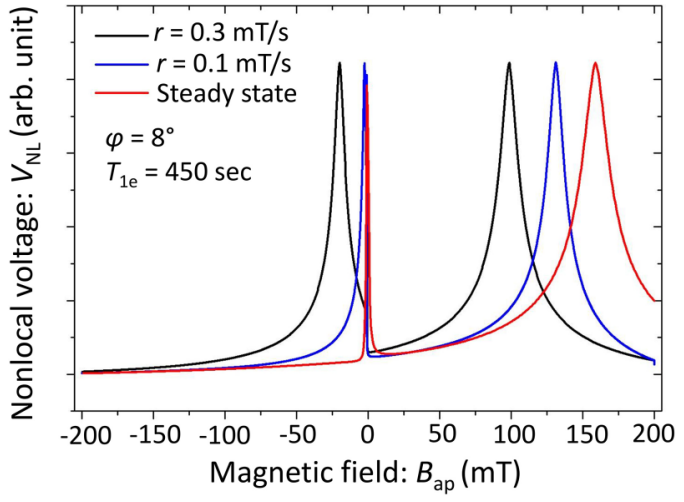

FIG. 5. Comparison of a steady-state solution of $V_{\mathrm{NL}}$ and transient solutions for $r=0.1$ and $0.3 \mathrm{mT} / \mathrm{s}$.

the solution of Eq. (2) converged to the steady-state one, $\mathbf{B}_{\mathbf{n} \mathbf{0}}$. Figure 5 compares the steady-state solution of $V_{\mathrm{NL}}$ and transient solutions for $r=0.1$ and $0.3 \mathrm{mT} / \mathrm{s}$. A dramatic difference between the transient and steady cases appears at state 5; the negative side peak disappears in the steadystate case. Since $\mathbf{B} \cdot \mathbf{S}_{\mathbf{0}}<0$ for the magnetic configuration in state $5, \mathbf{B}_{\mathbf{n} 0}$ and $\mathbf{B}$ are parallel. This indicates that no cancellation occurs for the steady state of state 5, resulting in the disappearance of the negative side peak. On the other hand, as $r$ increases, $\mathbf{B}_{\mathbf{n}}$ remains antiparallel to $\mathbf{B}$ even in state 5, as shown in Fig. 3. Thus, the $r$ dependence of the negative side peaks can be understood by the fact that the behaviors of the nuclear spins get closer to the steady state as $r$ decreases.

\section{Nuclear-spin-related phenomena appearing in spin-valve signals}

Figure 6(a) shows the experimental results of the spin-valve signal measurement for a fixed sweep rate $(r=0.5 \mathrm{mT} / \mathrm{s})$. The signal shows the change in $V_{\mathrm{NL}}$ as a function of the in-plane magnetic field $B_{\text {ap }}$, which was swept according to Eq. (A2) in Appendix A. The closed and open circles in the figure refer to positive and negative sweeps, respectively. As for the normal behavior of the spin-valve signal, $V_{\mathrm{NL}}$ drops to a lower value in $-30<B_{\text {ap }}<-24$ and $+25<B_{\text {ap }}<+29$ mT owing to the switching from parallel to antiparallel states for the magnetization configuration between the injector and detector contacts.

Our focus here is to explore the dip in $V_{\mathrm{NL}}$ occurring at $B_{\text {ap }}=0$ during the positive sweep [80 $<t \leqslant 240 \mathrm{~s}$ in Eq. (A2) in Appendix A], indicating a loss of electron-spin polarization under the detector [see Fig. 6(b)]. We simulated $V_{\mathrm{NL}}$ during the positive sweep direction for $B_{\text {ap }}$ from -10 to $10 \mathrm{mT}$ under the parallel magnetization configuration between the injector and detector contact. Our model fits the dip in $V_{\mathrm{NL}}$ at $B_{\text {ap }}=0$, as shown in Fig 6(b). The analysis evinces that the presence of this depolarization dip is due to the Hanle precession of electron spins induced by the transiently produced nuclear field. Figure 6(c) shows a vector diagram for the relative orientation of the injected electron spin $\mathbf{S}_{\mathbf{0}}$, magnetic field $\mathbf{B}$, and nuclear field $\mathbf{B}_{\mathbf{n}}$. The total magnetic field $\mathbf{B}$ rotates in the $x-z$ plane from $-\mathbf{x}$ to $+\mathbf{x}$ through the $+z$ direction. Since the time scale of the change in the strength of $\mathbf{B}_{\mathbf{n}}$, which is given by $\left(1 / T_{1 \mathrm{e}}+1 / T_{1}\right)^{-1}$, is much slower than that for the change in $\mathbf{B}, \mathbf{B}_{\mathbf{n}}$ rotates almost adiabatically as $\mathbf{B}$ rotates. In state $1 s$ in Fig. 6(c), $\mathbf{B}_{\mathbf{n}}$ is generated antiparallel to $\mathbf{B}$.

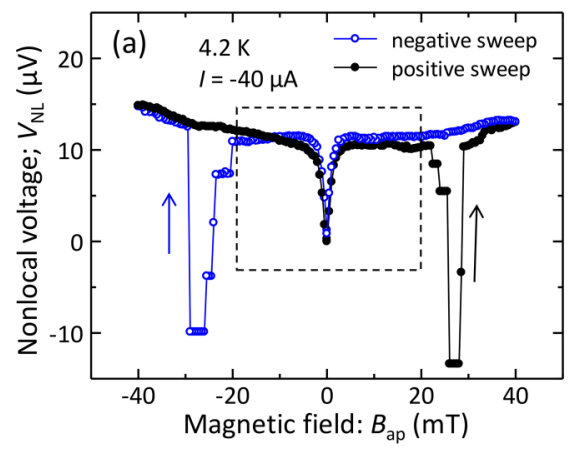

(c)
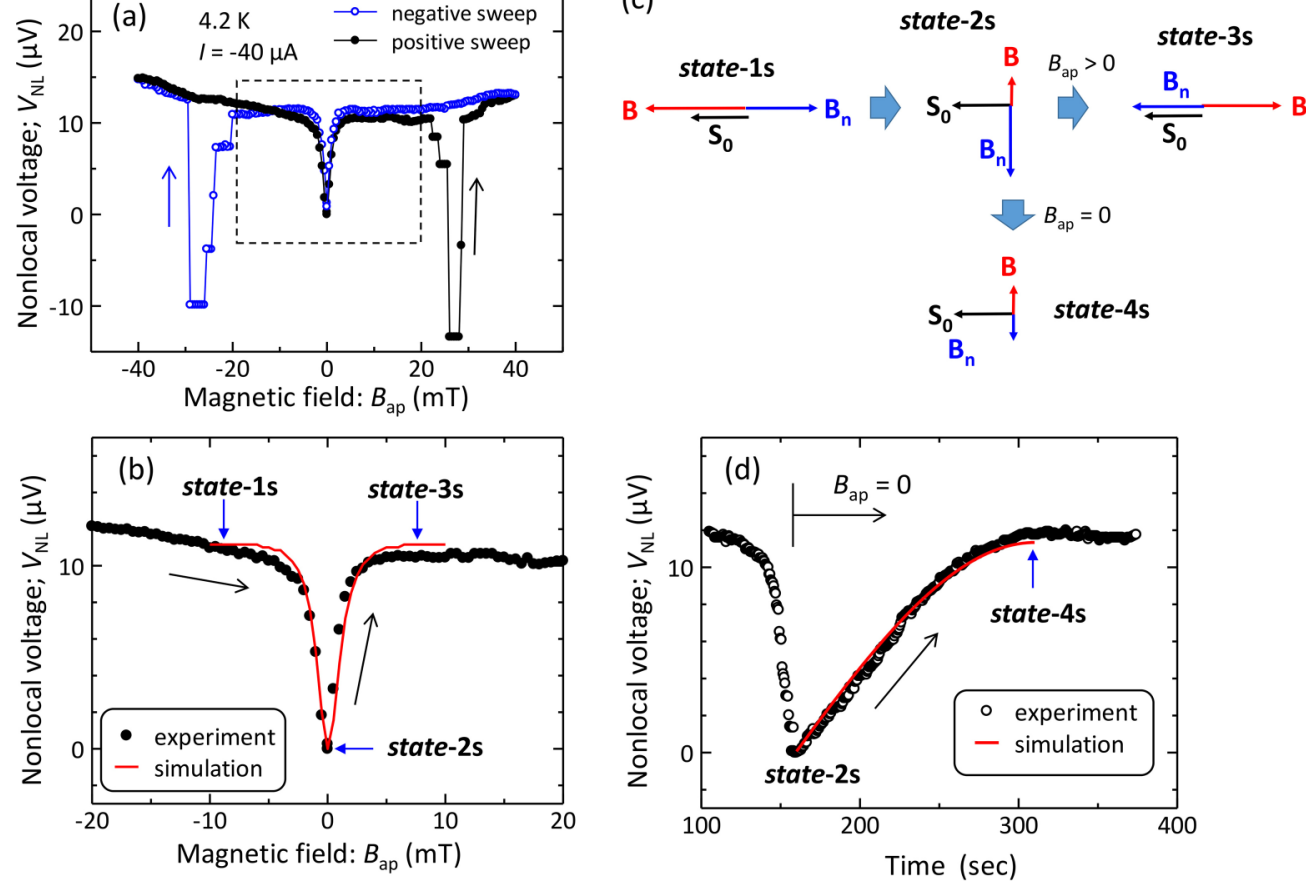

FIG. 6. (a) Experimental results of the nonlocal spin-valve signal measured at $4.2 \mathrm{~K}$ for a fixed bias current $I_{\text {bias }}=-40 \mu \mathrm{A}$. (b) Closed circles show the spin-valve signal for positive sweep only along with (red curve) fit with simulation result for parallel configuration. (c) Schematic vector diagrams showing relative orientations of $\mathbf{S}_{\mathbf{0}}, \mathbf{B}$, and $\mathbf{B}_{\mathbf{n}}$ in the spin-valve measurement. (d) $V_{\mathrm{NL}}$ as a function of time $(t)$. Open circles show the experimental result and the red curve indicates the simulation after $\mathbf{B}_{\text {ap }}$ was switched off at $t=160 \mathrm{~s}$. 
Since $\left(\mathbf{B}_{\mathbf{n}}+\mathbf{B}\right) \cdot \mathbf{S}_{\mathbf{0}}=0$, no electron-spin precession occurs, and $V_{\mathrm{NL}}$ equals $V_{\mathrm{NL}}^{\mathrm{P}}$, the original value of $V_{\mathrm{NL}}$ in the parallel magnetization configuration. In state $2 s, B_{\text {ap }}=0$; however, the transient $\mathbf{B}_{\mathbf{n}}$ still exists and is orthogonal to $\mathbf{S}_{\mathbf{0}}$ because of the presence of the stray field. Then, $V_{\mathrm{NL}}$ decreases from $V_{\mathrm{NL}}^{\mathrm{P}}$ as a result of the strong electron-spin precession. When $B_{\text {ap }}$ turns positive in state $3 s, V_{\mathrm{NL}}$ recovers to $V_{\mathrm{NL}}^{\mathrm{P}}$ again, because $\left(\mathbf{B}_{\mathbf{n}}+\mathbf{B}\right) \cdot \mathbf{S}_{\mathbf{0}}=0$, and the electron-spin precession is suppressed.

To further check that the $\operatorname{dip}$ in $V_{\mathrm{NL}}$ at $B_{\text {ap }}=0$ originated from the transiently produced nuclear field, we stopped the sweeping of the applied magnetic field exactly at $B_{\text {ap }}=0$ and continuously recorded $V_{\mathrm{NL}}$ [see Eq. (A3) in Appendix A]. The result of the recovery test of $V_{\mathrm{NL}}$ as a function of time is shown in Fig. 6(d), along with a simulation result. The $V_{\mathrm{NL}}$ recovered from its depolarization $\operatorname{dip}(=0 \mathrm{~V})$ to $V_{\mathrm{NL}}^{\mathrm{P}}$ and fitted well with the simulation result. Since the strength of $\mathbf{B}_{\mathbf{n}}$ decreases exponentially after switching off the magnetic field, whose time constant given by $\left(1 / T_{1 \mathrm{e}}+1 / T_{1}\right)^{-1}$ is approximately $72 \mathrm{~s}$ for $B_{\text {ap }}=0$, the nuclear field becomes negligibly small after a sufficient time ( $t>300 \mathrm{~s})$, as shown in state $4 \mathrm{~s}$ in Fig. 6(c).

Finally, let us discuss the effect of the stray field. According to Eq. (1), if $B_{\text {st }}=0$, then $T_{1}=0$ at $B_{\text {ap }}=0$. This indicates that the nuclear spins quickly relaxed to their thermal equilibrium at $B_{\text {ap }}=0$, and the nuclear-spin polarization disappeared. However, we experimentally found that the nuclear spins remained polarized for several hundred seconds after $B_{\text {ap }}$ reached 0, as shown in Figs. 2(a), 4(a), and 6(a)-6(d). Thus, an effective field which can keep the nuclear-spin polarization even at $B_{\text {ap }}=0$ should be present, and we assumed a constant stray field for such an effective field. Another possible origin of the effective field is the Knight field which acts on nuclear spins from polarized electron spins [13]. Further study, however, is necessary to clarify the origin for the effective field.

\section{SUMMARY AND CONCLUSIONS}

We systematically investigated the transient response of nuclear spins interacting with polarized electron spins through the hyperfine interaction in an $n$-GaAs channel through Hanle signal and spin-valve signal measurements in an all-electrical spin-injection device. Furthermore, we simulated Hanle and spin-valve signals by using the time evolution of the nuclear field by taking both $T_{1 \mathrm{e}}$ and $T_{1}$ into consideration. The simulation results reproduced the experimental results on all the nuclear-spin-related phenomena appearing in the Hanle and spin-valve signals, providing quantitative descriptions of the transient response of nuclear spins to fast changes in the magnetic field. Results showed that the direction of $\mathbf{B}_{\mathbf{n}}$ quickly rotated in space while reversing the direction of $\mathbf{B}$; however, its strength changed slowly with time depending on polarization and relaxation rates. The estimated value of $T_{1 \mathrm{e}}=450 \mathrm{~s}$ is reasonable as a time scale for the DNP in a GaAs semiconductor. Our findings provide not only a deep understanding of the spin physics in a semiconductor, but also useful information for achieving a precise control of nuclear spins.

\section{ACKNOWLEDGMENTS}

The authors would like to thank Prof. Masafumi Yamamoto, Prof. Kenji Kondo, Prof. Satoru Adachi, and Prof. Rena Kaji for their fruitful discussions. This work was supported by the Japan Society for the Promotion of Science (KAKENHI: Grants No. 25286039, No. 15K13960, No. 16K04872, and No. 17H03225).

\section{APPENDIX A: EXPERIMENTAL CONDITIONS FOR THE APPLIED MAGNETIC FIELD}

We summarize the sequences by which the applied magnetic field $B_{\text {ap }}$ changes as a function of time $t$ for both oblique Hanle signal and spin-valve signal measurements.

(1) The change of $B_{\text {ap }}$ as a function of $t$ for an oblique Hanle-signal measurement was as follows:

$$
B_{\text {ap }}(t)=\left\{\begin{array}{cc}
48 & \left(0 \leqslant t \leqslant t_{\text {hold }}\right) \\
48-r\left(t-t_{\text {hold }}\right) & \left(t_{\text {hold }}<t \leqslant t_{\text {hold }}+96 / r\right)
\end{array},\right.
$$

where $r$ is a sweep rate and $t_{\text {hold }}$ is the initialization time. The conditions for $t_{\text {hold }}$ and $r$ are summarized as follows:

(a) $t_{\text {hold }}=25,50,100$, and $150 \mathrm{~s}$ while fixing the sweep rate $r$ to $0.7 \mathrm{mT} / \mathrm{s}$, and

(b) $r=0.2,0.35,0.5$, and $0.7 / \mathrm{mT} / \mathrm{s}$ while fixing $t_{\text {hold }}$ at $100 \mathrm{~s}$.

(2) The change of $B_{\text {ap }}$ as function of $t$ for a spin-valve signal measurement was as follows:

Scheme 1. For the conventional spin-valve signal measurement

$$
B_{\text {ap }}(t)=\left\{\begin{array}{ll}
-0.5 t & (0 \leqslant t \leqslant 80) \\
-40+0.5(t-80) & (80<t \leqslant 240) \\
40-0.5(t-240) & (240<t \leqslant 320)
\end{array} .\right.
$$

Scheme 2. For the recovery test of central dip of $V_{\mathrm{NL}}$ appearing on spin-valve signal

$$
B_{\text {ap }}(t)=\left\{\begin{array}{ll}
-0.5 t & (0 \leqslant t \leqslant 80) \\
-40+0.5(t-80) & (80<t \leqslant 160) \\
0 & (160<t \leqslant 360)
\end{array} .\right.
$$

\section{APPENDIX B: DERIVATION OF EQS. (1)-(3)}

Here we describe how Eqs. (1)-(3) are derived together with the physics behind the DNP. The Hamiltonian of the Fermi-contact hyperfine interaction (HFI) is given by [52]

$$
H_{\mathrm{HFI}}=\frac{A}{2}\left(I_{+} S_{-}+I_{-} S_{+}\right)+A I_{z^{\prime}} S_{z^{\prime}},
$$

where $A$ is a coupling constant of the HFI, $I_{+(-)}$and $S_{+(-)}$are the raising (lowering) operators for nuclear spin and electron spin, respectively, and $I_{\mathrm{Z}^{\prime}}$ and $S_{\mathrm{Z}^{\prime}}$ are the components of the nuclear and electron spins along the magnetic field $\mathbf{B}$. The first term $\left(I_{+} S_{-}+I_{-} S_{+}\right)$of Eq. (B1) corresponds to the 
DNP through the flip-flop process, in which the nuclear spin and electron spin exchange their angular momentum through simultaneous reversal. The second term $\left(A I_{\mathrm{Z}^{\prime}} S_{\mathrm{Z}^{\prime}}\right)$, which is the static part of the hyperfine interaction, describes an effective magnetic field: an Overhauser field seen by electron spins or a Knight field seen by nuclear spins. In the mean-field approximation, the Overhauser field is given by [52]

$$
B_{\mathrm{n}}=A\left\langle I_{z^{\prime}}\right\rangle / g_{e} \mu_{\mathrm{B}},
$$

where $\left\langle I_{z^{\prime}}\right\rangle$ is the average spin of the polarized nuclei.

The time evolution of $\left\langle I_{z^{\prime}}\right\rangle$ is given by

$$
\frac{d}{d t}\left\langle I_{z^{\prime}}\right\rangle=-\frac{1}{T_{1 \mathrm{e}}}\left(\left\langle I_{z^{\prime}}\right\rangle-Q\left\langle S_{z^{\prime}}\right\rangle\right)-\frac{1}{T_{1}}\left\langle I_{z^{\prime}}\right\rangle,
$$

where $\left\langle S_{z^{\prime}}\right\rangle$ is the average electron spin, and $Q$ defines the momentum conversion coefficient from an electron-spin to a nuclear-spin system.

If $\left\langle I_{z^{\prime}}\right\rangle<Q\left\langle S_{z^{\prime}}\right\rangle$ for an initial condition, which is satisfied in most cases, then the first term of Eq. (B3) describes the nuclear-spin polarization through the flip-flop process. If we ignore the second term of Eq. (B3), $\left\langle I_{\mathrm{Z}^{\prime}}\right\rangle$ increases with a rate of $T_{1 \mathrm{e}}^{-1}$ until it reaches $Q\left\langle S_{\mathrm{z}^{\prime}}\right\rangle$, in which the dynamic processes of $I_{+} S_{-}$and $I_{-} S_{+}$are balanced. Owing to the large mismatch between electronic and nuclear Zeeman energies, those spinflip processes need some additional assisting processes, such as an emission or an absorption of photon or phonon in order to conserve an energy of the total spin systems. The nuclear polarization rate is, therefore, determined by the strength of the hyperfine coupling and the rate of the assisting process, and it is expressed as $[6,18]$

$$
T_{1 \mathrm{e}}^{-1}=\eta \tau_{1 \mathrm{e}}^{-1},
$$

where $\eta$ depends on the strength of the hyperfine coupling, and $\tau_{1 \mathrm{e}}^{-1}$ is the transition rate of spin flip-flop assisting process through the hyperfine interaction.

The second term of Eq. (B3), on the other hand, describes the nuclear-spin depolarization. The dipole-dipole interactions with neighboring nuclei $B_{\mathrm{L}}$ mix the wave functions of nuclear-spin states on the order of $B_{\mathrm{L}} /|\mathbf{B}|$. The nuclear-spin depolarization is led by the transitions between these mixed states, which is induced by the hyperfine magnetic field of electron spins $\left(\propto A\left\langle S_{\mathrm{Z}^{\prime}}\right\rangle\right)$. Then, the rate of nuclear-spin depolarization is given by $[6,18]$

$$
T_{1}^{-1}=\eta \tau_{1}^{-1}\left(\frac{B_{L}^{2}}{\mathbf{B}^{2}}\right)
$$

where $\tau_{1}^{-1}$ is the rate of the assisting depolarization process. From Eqs. (B4) and (B5), we get Eq. (1), where $\xi=\tau_{1 e} / \tau_{1}$. From the static solution of Eq. (B3) together with Eqs. (B2), (B4), and (B5), we get Eqs. (2) and (3).
[1] G. Lampel, Phys. Rev. Lett. 20, 491 (1968).

[2] A. I. Ekimov and V. I. Safarov, JETP Lett. 15, 319 (1972).

[3] M. I. D'yakonov, V. I. Perel', V. L. Berkovits, and V. I. Safarov, Sov. Phys. - JETP 40, 950 (1975).

[4] D. Paget, G. Lampel, B. Sapoval, and V. I. Safarov, Phys. Rev. B 15, 5780 (1977).

[5] S. E. Barrett, R. Tycko, L. N. Pfeiffer, and K. W. West, Phys. Rev. Lett. 72, 1368 (1994).

[6] D. Gammon, Al. L. Efros, T. A. Kennedy, M. Rosen, D. S. Katzer, D. Park, S. W. Brown, V. L. Korenev, and I. A. Merkulov, Phys. Rev. Lett. 86, 5176 (2001).

[7] G. Salis, D. T. Fuchs, J. M. Kikkawa, D. D. Awschalom, Y. Ohno, and H. Ohno, Phys. Rev. Lett. 86, 2677 (2001).

[8] M. Poggio, G. M. Steeves, R. C. Myers, Y. Kato, A. C. Gossard, and D. D. Awschalom, Phys. Rev. Lett. 91, 207602 (2003).

[9] P. L. Kuhns, A. Kleinhammes, T. Schmiedel, W. G. Moulton, P. Chabrier, S. Sloan, E. Hughes, and C. R. Bowers, Phys. Rev. B 55, 7824 (1997).

[10] H. Sanada, Y. Kondo, S. Matsuzaka, K. Morita, C. Y. Hu, Y. Ohno, and H. Ohno, Phys. Rev. Lett. 96, 067602 (2006).

[11] J. Lu, M. J. R. Hoch, P. L. Kuhns, W. G. Moulton, Z. Gan, and A. P. Reyes, Phys. Rev. B 74, 125208 (2006).

[12] S. Mui, K. Ramaswamy, and S. E. Hayes, Phys. Rev. B 75, 195207 (2007).

[13] R. Matsusaki, R. Kaji, S. Yamamoto, H. Sasakura, and S. Adachi, arXiv:1703.06046.

[14] T. Machida, T. Yamazaki, and S. Komiyama, Appl. Phys. Lett. 80, 4178 (2002).
[15] K. Hashimoto, K. Muraki, T. Saku, and Y. Hirayama, Phys. Rev. Lett. 88, 176601 (2002).

[16] J. Strand, B. D. Schultz, A. F. Isakovic, C. J. Palmstrøm, and P. A. Crowell, Phys. Rev. Lett. 91, 036602 (2003).

[17] K. Ono and S. Tarucha, Phys. Rev. Lett. 92, 256803 (2004).

[18] J. Strand, X. Lou, C. Adelmann, B. D. Schultz, A. F. Isakovic, C. J. Palmstrøm, and P. A. Crowell, Phys. Rev. B 72, 155308 (2005).

[19] P. Van Dorpe, W. Van Roy, J. De Boeck, and G. Borghs, Phys. Rev. B 72, 035315 (2005).

[20] M. K. Chan, Q. O. Hu, J. Zhang, T. Kondo, C. J. Palmstrøm, and P. A. Crowell, Phys. Rev. B 80, 161206(R) (2009).

[21] G. Salis, A. Fuhrer, and S. F. Alvarado, Phys. Rev. B 80 , 115332(R) (2009).

[22] J. Shiogai, M. Ciorga, M. Utz, D. Schuh, T. Arakawa, M. Kohda, K. Kobayashi, T. Ono, W. Wegscheider, D. Weiss, and J. Nitta, Appl. Phys. Lett. 101, 212402 (2012).

[23] T. Akiho, J. Shan, H.-x. Liu, K.-i. Matsuda, M. Yamamoto, and T. Uemura, Phys. Rev. B 87, 235205 (2013).

[24] T. Uemura, T. Akiho, Y. Ebina, and M. Yamamoto, Phys. Rev. B 91, 140410(R) (2015).

[25] Z. Lin, M. Rasly, and T. Uemura, Appl. Phys. Lett. 110, 232404 (2017).

[26] K. Inomata, S. Okamura, R. Goto, and N. Tezuka, Jpn. J. Appl. Phys. 42, L419 (2003).

[27] Y. Sakuraba, M. Hattori, M. Oogane, Y. Ando, H. Kato, A Sakuma, T. Miyazaki, and H. Kubota, Appl. Phys. Lett. 88 192508 (2006).

[28] T. Ishikawa, H.-x. Liu, T. Taira, K.-i. Matsuda, T. Uemura, and M. Yamamoto, Appl. Phys. Lett. 95, 232512 (2009). 
[29] N. Tezuka, N. Ikeda, F. Mitsuhashi, and S. Sugimoto, Appl. Phys. Lett. 94, 162504 (2009).

[30] W. Wang, E. Liu, M. Kodzuka, H. Sukegawa, M. Wojcik, E. Jedryka, G. H. Wu, K. Inomata, S. Mitani, and K. Hono, Phys. Rev. B 81, 140402(R) (2010).

[31] M. Yamamoto, T. Ishikawa, T. Taira, G.-f. Li, K.-i. Matsuda, and T. Uemura, J. Phys.: Condens. Matter 22, 164212 (2010).

[32] H.-x. Liu, Y. Honda, T. Taira, K.-i. Matsuda, M. Arita, T. Uemura, and M. Yamamoto, Appl. Phys. Lett. 101, 132418 (2012).

[33] H.-x. Liu, T. Kawami, K. Moges, T. Uemura, M. Yamamoto, F. Shi, and P. M. Voyles, J. Phys. D: Appl. Phys. 48, 164001 (2015).

[34] K. Moges, Y. Honda, H.-x. Liu, T. Uemura, M. Yamamoto, Y. Miura, and M. Shirai, Phys. Rev. B 93, 134403 (2016).

[35] B. Hu, K. Moges, Y. Honda, H.-x. Liu, T. Uemura, M. Yamamoto, J.-i. Inoue, and M. Shirai, Phys. Rev. B 94, 094428 (2016).

[36] K. Yakushiji, K. Saito, S. Mitani, K. Takanashi, Y. K. Takahashi, and K. Hono, Appl. Phys. Lett. 88, 222504 (2006).

[37] T. Furubayashi, K. Kodama, H. Sukegawa, Y. K. Takahashi, K. Inomata, and K. Hono, Appl. Phys. Lett. 93, 122507 (2008).

[38] Y. Sakuraba, K. Izumi, T. Iwase, S. Bosu, K. Saito, K. Takanashi, Y. Miura, K. Futatsukawa, K. Abe, and M. Shirai, Phys. Rev. B 82, 094444 (2010).

[39] Y. K. Takahasi, A. Srinivasan, B. Varaprasad, A. Rajanikanth, N. Hase, T. M. Nakatani, S. Kasai, T. Furubayashi, and K. Hono, Appl. Phys. Lett. 98, 152501 (2011).

[40] H. Narisawa, T. Kubota, and K. Takanashi, Appl. Phys. Express 8, 063008 (2015).

[41] J. W. Jung, Y. Sakuraba T. T. Sasaki, Y. Miura, and K. Hono, Appl. Phys. Lett. 108, 102408 (2016).
[42] M. Inoue, B. Hu, K. Moges, K. Inubushi, K. Nakada, M. Yamamoto, and T. Uemura, Appl. Phys. Lett. 111, 082403 (2017).

[43] X. Y. Dong, C. Adelmann, J. Q. Xie, C. J. Palmstrøm, X. Lou, J. Strand, P. A. Crowell, J. Barnes, and A. K. P.-Long, Appl. Phys. Lett. 86, 102107 (2005).

[44] P. Bruski, Y. Manzke, R. Farshchi, O. Brandt, J. Herfort, and M. Ramsteiner, Appl. Phys. Lett. 103, 052406 (2013).

[45] T. Saito, N. Tezuka, M. Matsuura, and S. Sugimoto, Appl. Phys. Express 6, 103006 (2013).

[46] Y. Ebina, T. Akiho, H.-x. Liu, M. Yamamoto, and T. Uemura, Appl. Phys. Lett. 104, 172405 (2014).

[47] T. A. Peterson, S. J. Patel, C. C. Geppert, K. D. Christie, A. Rath, D. Pennachio, M. E. Flatte, P. M. Voyles, C. J. Palmstrøm, and P. A. Crowell, Phys. Rev. B 94, 235309 (2016).

[48] M. Yamada, M. Tsukahara, Y. Fujita, T. Naito, S. Yamada, K. Sawano, and K. Hamaya, Appl. Phys. Exp. 10, 093001 (2017).

[49] J. Kübler, A. R. Williams, and C. B. Sommers, Phys. Rev. B 28, 1745 (1983).

[50] S. Ishida, S. Fujii, S. Kashiwagi, and S. Asano, J. Phys. Soc. Jpn. 64, 2152 (1995).

[51] S. Picozzi, A. Continenza, and A. J. Freeman, Phys. Rev. B 66, 094421 (2002).

[52] M. I. Dyakonov and V. I. Perel, Optical Orientation, edited by F. Meier and B. P. Zakharchenya (North-Holland, New York, 1984).

[53] Z. Lin, K. Kondo, M. Yamamoto, and T. Uemura, Jpn. J. Appl. Phys., Part 1 55, 04EN03 (2016).

[54] M. Rasly, Z. Lin, M. Yamamoto, and T. Uemura, AIP Adv. 6, 056305 (2016).

[55] J. Fabian, A. Matos-Abiague, C. Ertler, P. Stano, and I. Žutić, Acta Phys. Slovaca 57, 565 (2007). 